\title{
"In-silico Prediction of Riboswitches and Design of their Potent Inhibitors for H1N1, H2N2 and H3N2 Strains of Influenza Virus"
}

\author{
Priya Pradhan*, Nishant K. Soni, Lalitesh Chaudhary, \\ Somdutt Mujwar and K.R. Pardasani
}

Department of Bioinformatics, Maulana Azad National Institute of Technology, Bhopal 462 051, India.

DOI: http://dx.doi.org/10.13005/bbra/1889

(Received: 15 August 2015; accepted: 21 September 2015)

\begin{abstract}
Almost every age group is at higher risk for serious flu complications. The major problem arising these days regarding the control of influenza disease is the development of resistance among the influenza viruses against the existing anti-viral drugs that are being recommended. Also, these antiviral drugs have a number of side effects. The main objective of the present paper is to explore riboswitches as a novel target for design of drugs for influenza virus in order to address the issues of resistance and side effects of present drugs. Riboswitches are present in the non-coding region of mRNA that sense changes in the cellular environment and directly mediate appropriate gene control responses. These riboswitches are primarily found in the 5' untranslated regions of messenger RNAs. In the present paper in-silico approach is proposed for the prediction of riboswitches for the strains of influenza virus, their binding sites and design of their inhibitors. Two riboswitches have been predicted for the three strains of influenza virus and five inhibitors have been identified for each of the two riboswitches by virtual screening. These inhibitors are found to be free from the side effects of antiviral agents and have remote chances of being resistant.
\end{abstract}

Key words: Riboswitches, Influenza, Inhibitors, AutoDock, Drug, Virtual Screening

Influenza is a contagious disease affecting the respiratory tract, caused by Influenza viruses A, B, C mainly A, its symptoms can lead from mild to severe illness and at times can lead to death as compared to typical sneezing and stuffiness of common cold. The flu viruses spread mainly by droplets when people with the flu cough, sneeze or talk. These droplets mainly land in the mouths or noses of people who are in the vicinity ${ }^{1-}$ ${ }^{7}$. Moreover, the flu may get spread over an area directly or indirectly.

Symptoms of Influenza are Fever, Cough, Sore throat, Runny nose, Muscle or body aches,

\footnotetext{
* To whom all correspondence should be addressed.
}

Headaches, Fatigue and some people may have vomiting and diarrhea also ${ }^{8,9}$. Centers for Disease Control and Prevention estimated that around 90\% of seasonal flu related deaths and more than $60 \%$ of hospitalizations in the united states occur in people of 65 years of age and older because human immune system becomes weaker with age ${ }^{10}$.

People suffering with asthma have swollen and sensitive airways, and when they experience symptoms of influenza, it can cause further inflammation of the airways and lungs, not only asthmatic patients but also people with neurological disorders ${ }^{11}$ suffer badly with influenza. People suffering with chronic lung diseases, Blood disorders (such as sickle cell disease), Endocrine disorders (such as diabetes mellitus), Kidney disorders, Liver disorders, people with HIV or AIDS 
and heart disease are also at high risk for flu complications.

The major problem with the existing antiviral drugs is the development of resistance among the influenza viruses against these drugs. Earlier Adamantanes i.e. Amantadine and Rimantadine were given for influenza treatment, but it was observed that resistance among influenza a viruses increased so fast for Adamantanes that it became necessary to stop their use in the treatment. It was observed that Adamantane resistance increased from $0.4 \%$ during $1994-1995$ to $12.3 \%$ during 2003-2004 ${ }^{12}$.

After this treatment Neuraminidase Inhibitors i.e. Oseltamivir or zanamivir that are the primary antiviral agents were recommended for the prevention and treatment of influenza viruses ${ }^{13-15}$. But later the development of resistance ${ }^{16-19}$ for these antiviral drugs was reported which states that oseltamivir-resistant seasonal influenza A viruses were isolated from nine $(18 \%)$ out of 50 Japanese children during treatment ${ }^{17,20}$. Zanamivir is not recommended for immune-compromised children $^{16,21}$. During the post marketing surveillance allergic reactions, facial edema, and swelling are also reported ${ }^{22,23}$. Apart from the development of resistance, the antiviral drugs have number of side effects.

The major problem faced with vaccination i.e. flu shots is that it might have some of the common local adverse reactions that includes erythema (redness), induration (firmness), swelling, pain, and pruritus (itching) at the vaccination site; headache, myalgia (muscle ache), and malaise.

These issues of drugs resistance and side effects of existing drugs are of major concern for treatment of influenza virus and there thus there is a need to explore novel drugs targets and design new inhibitor for influenza virus. The riboswitches can be explored as new drug target for such infectious diseases.

Riboswitches are present in the noncoding region of mRNA that sense changes in the cellular environment and directly mediate appropriate gene control responses. These riboswitches are primarily found in the 5, untranslated regions of messenger RNAs. They are being divided into two parts- Evolutionary conserved sensor domain (an aptamer) which directly binds small molecules and an expression platform which undergoes structural changes in response to changes in aptamer ${ }^{24}$. Binding domains are highly conserved, even among divergent organisms. It is believed to have persisted through evolution. The Expression Platform Sequences vary widely among riboswitches, sequence even varies within a class. These parts of riboswitches in combination acts like the operon system which is based on the ON and OFF mechanism for the expression of the genes associated when the aptamer region is bound to the appropriate ligand to show the ligand's efficiency onto the specific riboswitch binding site.

The most common mechanisms of riboswitches include: (1) formation of the hairpin structure that leads to RNA polymerase stalling and premature transcription termination, (2) basepairing between Shine-Dalgarno and anti-ShineDalgarno sequence that blocks translation, and (3) changing the splice sites $^{25,26}$. Riboswitches as a novel drug target and identify its potential inhibitor for H1N1, H2N2 and H3N2 strains of influenza virus.

BioRelix Inc. is building a club of antiinfective drug treatments that target riboswitches, or stretches of messenger RNAs that control genes expression and mechanism essential to the survival of many disease-causing microbes. This strategy has applications in the elimination of pathogens that are resistant to currently available drugs.

\section{MATERIAL \& METHODS}

The proposed framework for in-silico prediction and identification of riboswitches, their ligands and its inhibitors are given below:-

1) Identification of Gene Sequence for Riboswitch.

2) Human Nucleotide Database Search for Gene Sequence Similarity by BLAST

3) Transcription of Gene Sequence to Corresponding Riboswitch like Element (RLE).

4) Prediction of Structure of Riboswitch like Elements.

5) Blind Docking (BD).

6) Focused Docking (FD) for Identification of Ligand.

7) Refinement of Docking Results.

8) Virtual Screening Using NCI Diversity Set.

9) Evaluation of Physicochemical Properties 
of Lead Compounds.

10) Prediction of ADME \& Toxicity of Lead Compounds.

The flow chart of framework is given in Fig. 1. Identification of Gene Sequence for Riboswitch The whole genomes of $\mathrm{H} 1 \mathrm{~N} 1, \mathrm{H} 2 \mathrm{~N} 2$ and H3N2 Influenza virus are obtained from the National Center for Biotechnology Information (NCBI) Gene Bank in FASTA format. The viral genome is searched for riboswitch like sequences present in it by online program Riboswitch Explorer to identify Riboswitch like sequences ${ }^{6}$.

Human Nucleotide Database Search for Gene Sequence Similarity by BLAST

The viral riboswitch like gene sequences identified by RibEx are searched in the National Center for Biotechnology Information database for similar gene sequence present in Human (Homo Sapiens) genome by using Basic Linear Alignment Sequencing tool (BLAST).

Transcription of Gene Sequence to Corresponding Riboswitch like Element (RLE)

The gene sequences identified by online program RibEx for riboswitch like elements are transcripted to its corresponding riboswitch by using online "Transcription and Translation Tool". Structure Prediction of Riboswitch

The 3-Dimensional structure of the riboswitch like elements is predicted by using online program iFoldRNA. iFoldRNA program performs interactive RNA folding simulations using discrete molecular dynamics simulations using coarsegrained structural RNA models to predict the 3D structure of the identified sequence of riboswitch like elements present in the genomes of $\mathrm{H} 1 \mathrm{~N} 1, \mathrm{H} 2 \mathrm{~N} 2$ and H3N2 Influenza virus.

\section{Blind Docking (BD)}

AutoDock based Blind docking technique is used to find out appropriate binding site present in the predicted riboswitch like structures of $\mathrm{H} 1 \mathrm{~N} 1$, $\mathrm{H} 2 \mathrm{~N} 2$ and H3N2 Influenza virus. In blind docking technique whole structure of riboswitch is covered under imaginary $3 \mathrm{D}$ grid box for docking. The coordinates of grid box used for blind docking technique for all the two identified riboswitches PRI1 and PRI2 are given in Table-1. 27,10,4,28

All computational studies are carried out using Autodock 4.2 installed on a single machine running on a $2.80 \mathrm{GHz}$ Intel core 2 duo processor with 3 GB RAM and 320 GB hard disk with Windows
$\mathrm{XP}$ as an operating system.

Docking software's like "AutoDock Tools", "Autogrid" and "Autodock-4.2" were downloaded from the Scripps portal (http:// autodock.scripps.edu). AutoDock software explores the whole surface of riboswitch for binding of ligand in the riboswitch. Alanine (ALA) is used as a ligand in blind docking technique to identify binding sites present in all the two predicted riboswitches, as it is the neutral and simplest ligand out of possible 20 amino acids as substrate of a riboswitch. Appropriate binding sites present in the surface of the predicted riboswitches are identified on the basis of lowest binding energy in the range of -5 to $-15 \mathrm{Kcal} / \mathrm{Mol}$. The appropriate binding site present in the predicted riboswitches of influenza is used for identification of binding residues involved in binding of ligand. The identified binding residues present in the predicted riboswitches are further utilized for focused docking.

Focused Docking (FD) for Identification of Ligand

Focused grid box covering ligand as well as binding residues involved in binding of ligand, is prepared for focused docking targeting specific ligand binding site for all the two identified riboswitches of Influenza virus ${ }^{29-31}$. The coordinates containing the information about the size and position of grid box used in focused docking of all the two predicted influenza riboswitches are tabulated in Table-2. These three grid boxes are further utilized for focused docking with 20 different amino acids for identification of specific substrate ligand for all the two predicted riboswitches.

\section{Refinement of Docking Results}

After identification of an appropriate ligand for predicted riboswitches by focused docking, separate docking of identified substrate ligand with its corresponding riboswitch is done for all the two riboswitches identified in the genome of Influenza virus repeatedly for a number of times to refine the results.

\section{Virtual Screening Using NCI Diversity Set}

The identified binding sites present in all the two predicted structures of novel Influenza riboswitches are utilized for virtual screening of NCI Diversity Set containing 1541 diverse molecules $^{32}$. All the files necessary for virtual screening are prepared by software Raccoon. It is 
a graphical user interface for AutoDock virtual screening (autodock.scripps.edu/resources/ raccoon). Raccoon can split multiple-molecule ligand library files, convert them into the AutoDock format (i.e. *. pdbqt), and filter them by using common criteria (e.g., Lipinski's rules, fragmentlike "rule of 3", and drug-likeness). A validation check of the input files is performed at every step, which includes checking for the presence of nonstandard atom types and ensuring that parameters, input file names, and grid maps have a coherent format.

Molecular docking simulation based virtual screening of all the two influenza riboswitches is done using similar docking and grid parameters used in focused docking earlier.

The coordinates of the grid box used in the virtual screening process of molecular libraries containing 1880 molecules against the binding site of riboswitches are tabulated in Table-3. The coordinates of grid box used in virtual screening of both the riboswitches PRI1 and PRI2 are given in Table-3.

\section{Evaluation of Physicochemical Properties of Lead Compounds}

The top five screened ligands for each riboswitch are evaluated for important physicochemical properties such as calculated partition coefficient (ClogP), 2D-Polar surface area (2D PSA), molecular weight, hydrogen bond donor and acceptor sites etc. by using Marvin Sketch software.

Prediction of ADME \& Toxicity of Lead Compounds

The top 5 lead molecules for both the two riboswitches are evaluated by using the OSIRIS online program for toxicity and ADME properties ${ }^{33}$. This program evaluates the presence of major toxicities such as mutagenicity, tumorigenicity, irritant effect and reproductive effects in the lead molecules on the basis of functional group present in their chemical structure. This program also calculates drug-likeness and drug score of the lead molecules on the basis of their physicochemical properties.

The results obtained in each of the above steps are presented in the next section.

\section{RESULTSAND DISCUSSION}

The stepwise results obtained are summarized and discussed below:

Identified Genes for Riboswitch like Sequence

The following two gene sequences are identified by the RibEx for transcription into riboswitch like elements present in the viral DNA of H1N1, H2N2 and H3N2 Influenza virus genome. PRI1- TATGAGGCCCATACAACTGGCAAGTG CACCAGCAGAATAA

PRI2-ATCCCAAAATCCCCTTAGTCAGAGG

Gene Sequence Similarity with Human Genome

The identified riboswitch like gene

Table 1. The coordinates of grid box for the Blind Docking (BD)

\begin{tabular}{lccccccc}
\hline Proteins & $x-D$ & $y-D$ & $z-D$ & Spacing $(\AA)$ & $x$ center & $y$ center & z center \\
\hline PRI1 & 74 & 48 & 44 & 0.803 & -0.295 & 0.931 & 2.852 \\
PRI2 & 58 & 60 & 62 & 0.603 & 2.447 & -0.951 & 0.900 \\
\hline
\end{tabular}

Table 2. The coordinates of grid box for the Focussed Docking (FD)

\begin{tabular}{lccccccc}
\hline Proteins & $\mathrm{x}-\mathrm{D}$ & $\mathrm{y}-\mathrm{D}$ & $\mathrm{z}-\mathrm{D}$ & $\mathrm{Spacing}(\AA)$ & $\mathrm{x}$ center & $\mathrm{y}$ center & $\mathrm{z}$ center \\
\hline PRI1 & 40 & 30 & 36 & 0.303 & 14.085 & -8.772 & -9.892 \\
PRI2 & 46 & 38 & 44 & 0.308 & 3.623 & 0.654 & 7.768 \\
\hline
\end{tabular}

Table 3. The coordinates of grid box for the Virtual Screening (VS)

\begin{tabular}{lccccccc}
\hline Proteins & $x-D$ & $y-D$ & $z-D$ & Spacing $(\AA)$ & $x$ center & $y$ center & $z$ center \\
\hline PRI1 & 40 & 30 & 36 & 0.303 & 14.085 & -8.772 & -9.892 \\
PRI2 & 46 & 38 & 44 & 0.308 & 3.623 & 0.654 & 7.768 \\
\hline
\end{tabular}


sequences of H1N1, H2N2 and H3N2 Influenza Virus shows the following results on the NCBI nucleotide database search for gene sequence similarity present in human (Homo sapiens) genome.

\section{PRI1}

The gene sequence of PRI1 riboswitch shows a BLAST score 38.2 with a maximum $97 \%$ of gene sequence similarity present in the human genome. The BLAST result of gene sequence similarity to PRI1 gene sequence is shown in Fig. 2(A). The human genome showing 38.2 blast score is searched for the presence of any riboswitch like gene sequence by using RibEx software. No riboswitch was found in similar gene in the human genome

Table 4. Focused Docking Results for PRI1

\begin{tabular}{llcc}
\hline $\begin{array}{l}\text { S. } \\
\text { No. }\end{array}$ & $\begin{array}{l}\text { Amino } \\
\text { acid }\end{array}$ & $\begin{array}{c}\text { Binding Energy } \\
\text { (Kcal/Mol) }\end{array}$ & Ki $(\mu \mathrm{M})$ \\
\hline 1. & ALA & -5.24 & 144.21 \\
2. & ARG & -6.22 & 27.39 \\
3. & ASN & -5.61 & 77.27 \\
4. & ASP & -5.5 & 93.13 \\
5. & CYS & -5.15 & 166.65 \\
6. & GLN & -6.18 & 29.62 \\
7. & GLU & -6.12 & 32.44 \\
8. & GLY & -5.06 & 196.66 \\
9. & HIS & -5.02 & 207.94 \\
10. & ILE & -5.68 & 68.27 \\
11. & LEU & -5.8 & 55.72 \\
12. & LYS & -7.26 & 4.74 \\
13. & MET & -5.21 & 151.69 \\
14. & PHE & -5.29 & 132.87 \\
15. & PRO & -4.95 & 237.24 \\
16. & SER & -5.69 & 67.11 \\
17. & THR & -5.97 & 41.82 \\
18. & TRP & -5.57 & 82.57 \\
19. & TYR & -5.21 & 151.98 \\
20. & VAL & -5.9 & 47.45 \\
\hline
\end{tabular}

Table 6. Blind Docking Results

\begin{tabular}{lccc} 
S. No. & Ligand & $\begin{array}{c}\text { Binding Energy } \\
(\mathrm{Kcal} / \mathrm{Mol})\end{array}$ & $\mathrm{Ki}(\mu \mathrm{M})$ \\
\hline PRI1 & ALA & -5.24 & 144.21 \\
PRI2 & ALA & -5.05 & 199.83 \\
\hline
\end{tabular}

PRI2

The gene sequence of SPR1 riboswitch shows a BLAST score 34.2 with a maximum $72 \%$ gene sequence similarity present in the human genome. The BLAST result of gene sequence similarity to PRI2 gene sequence is shown in Fig 2(B). The human genome showing 34.2 blast score is searched for the presence of any riboswitch like gene sequence by using RibEx software. No riboswitch was found in similar gene in the human genome.

\section{Transcription of Gene Sequence to Riboswitch like} Element Sequence

Following sequences are identified after transcription of identified gene sequences of Riboswitch like elements;

PRI1 - UAUGAGGCCCAUACAACUGGCAAG UGCACCAGCAGAAUAA

PRI2-AUCCCAAAAUCCCCUUAGUCAGAGG

Table 5. Focused Docking Results for PRI2

\begin{tabular}{llcc}
\hline $\begin{array}{l}\text { S. } \\
\text { No. }\end{array}$ & $\begin{array}{l}\text { Amino } \\
\text { acid }\end{array}$ & $\begin{array}{c}\text { Binding Energy } \\
(\text { Kcal/Mol })\end{array}$ & Ki $(\mu \mathrm{M})$ \\
\hline 1. & ALA & -5.05 & 199.83 \\
2. & ARG & -6.37 & 21.28 \\
3. & ASN & -6.23 & 27.08 \\
4. & ASP & -3.7 & 1940 \\
5. & CYS & -5.29 & 133.44 \\
6. & GLN & -5.61 & 77.41 \\
7. & GLU & -4.2 & 840.94 \\
8. & GLY & -4.88 & 264.43 \\
9. & HIS & -6.13 & 32.32 \\
10. & ILE & -5.64 & 72.83 \\
11. & LEU & -5.69 & 67.4 \\
12. & LYS & -6.33 & 23.02 \\
13. & MET & -5.37 & 115.58 \\
14. & PHE & -6.11 & 33.07 \\
15. & PRO & -4.66 & 382.37 \\
16. & SER & -5.35 & 119.04 \\
17. & THR & -5.6 & 78.58 \\
18. & TRP & -6.77 & 10.91 \\
19. & TYR & -6.19 & 29.22 \\
20. & VAL & -5.44 & 103.33 \\
& & &
\end{tabular}

Table 7. Refined Docking Results

\begin{tabular}{lcccc}
\hline $\begin{array}{l}\text { S. } \\
\text { No }\end{array}$ & Riboswitch & Ligand & $\begin{array}{c}\text { Binding Energy Ki } \\
(\text { Kcal/Mol })\end{array}$ & $(\mu \mathrm{M})$ \\
\hline 1 & & & - & - \\
2 & PRI1 & ALA & - & - \\
\hline
\end{tabular}


Table 8. List of Binding Energies of Proposed lead molecules for PRI1

\begin{tabular}{|c|c|c|c|c|}
\hline S.No. & ID & Chemical Structure & $\begin{array}{l}\text { Binding Energy } \\
\qquad(\mathrm{kcal} / \mathrm{mol})\end{array}$ & $\begin{array}{l}\text { Binding affinity } \\
\qquad(\mu \mathrm{M})\end{array}$ \\
\hline 1 & NCI_81462_a & & -10.06 & 0.04193 \\
\hline 2 & NCI_168184 & & -7.95 & 1.49 \\
\hline 3 & NCI_113486 & & -7.53 & 3.01 \\
\hline 4 & NCI_207895 & & -7.21 & 5.15 \\
\hline 5 & NCI_3076_a & & -7.09 & 6.32 \\
\hline
\end{tabular}


Table 9. List of Binding Energies of Proposed lead molecules for PRI2

\begin{tabular}{|c|c|c|c|c|}
\hline $\begin{array}{l}\text { S. } \\
\text { No. }\end{array}$ & ID & Chemical Structure & $\begin{array}{l}\text { Binding Energy } \\
(\mathrm{kcal} / \mathrm{mol})\end{array}$ & $\begin{array}{c}\text { Binding affinity } \\
\text { (nM) }\end{array}$ \\
\hline 1 & NCI_117268_a & $-N$ & -9.56 & 97.57 \\
\hline 2 & NCI_170621 & & -9.16 & 193.71 \\
\hline 3 & NCI_281816_a & & -8.95 & 274.00 \\
\hline 4 & NCI_403379 & & -8.82 & 341.84 \\
\hline 5 & NCI_13316_b & & -8.46 & 633.76 \\
\hline
\end{tabular}


The identified two DNA gene sequences are transcripted to corresponding riboswitch like elements using online, "Transcription and Translation Tool". The tool replaces the thymine (T) nucleotide of the gene sequence with uracil (U) to form its corresponding riboswitch (mRNA). Predicted tertiary Structures of Riboswitch

The tertiary structures of both the identified riboswitches in $\mathrm{H} 1 \mathrm{~N} 1, \mathrm{H} 2 \mathrm{~N} 2$ and $\mathrm{H} 3 \mathrm{~N} 2$ Influenza virus are predicted by online program iFoldRNA, and following results are obtained.

(a) The tertiary structure of Influenza riboswitch PRI1 is identified by using online program iFoldRNA. The PRI1 riboswitch consists of a single chain of 40 nucleotides. The predicted tertiary structure of PRI1 riboswitch in Influenza virus is shown in Fig. 3.

(b) The tertiary structure of the Influenza riboswitch PRI2 is identified by using online program iFoldRNA. The PRI2 riboswitch consists of a single chain of 25 nucleotides. The predicted tertiary structure of PRI2 riboswitch in Influenza bacteria is shown in Fig. 4.

\section{Blind and Focused Docking Results}

(a) The binding site present in the Influenza riboswitch PRI1 identified by Blind docking suggests that the residues ADE34, ADE31
CYT33, GUA24 and GUA32 of PRI1 riboswitch are involved in the binding of ligand.

The focused docking of PRI1 riboswitch using the identified binding residues involved in the binding of the ligand with the riboswitch suggests the Lysine as its substrate ligand as it shows best binding among 20 amino acids with a binding energy value of $-7.26 \mathrm{Kcal} / \mathrm{Mol}(\mathrm{Ki}=4.74 \mu \mathrm{M})$. The ligand binding site identified in predicted three dimensional structure of PRI1 riboswitch of Influenza virus is shown in Fig. 6.

The focused docking result of PRI1 riboswitch is tabulated in Table-4.

(b) The binding site present in the Influenza riboswitch PRI2 is identified by blind docking suggests that the residues CYT11, URI10, ADE21, GUA22 and ADE23 of PRI2 riboswitch are involved in the binding of ligand.

The focused docking of PRI2 riboswitch using the identified binding residues involved in the binding of the ligand with the riboswitch suggests the Tryptophan as its substrate ligand as it shows best binding among 20 amino acids with a binding energy value of $-6.77 \mathrm{Kcal} / \mathrm{Mol}(\mathrm{Ki}$ $=10.91 \mu \mathrm{M})$. The ligand binding site identified in

Table 10. Physiochemical Parameters of Proposed lead molecules for PRI1 Riboswitch

\begin{tabular}{lcccccc}
\hline Parameter & $\begin{array}{c}\text { Optimum } \\
\text { range }\end{array}$ & NCI_81462_a & NCI_168184 & NCI_113486 & NCI_207895 & NCI_3076_a \\
\hline ClogP & -5 to +5 & 0.37 & 0.22 & -0.21 & -2.26 & 1.44 \\
PSA (2D) $\left(\AA^{2}\right)$ & 60 to 140 & 39.33 & 106.02 & 80.58 & 101 & 50.39 \\
Mol. Wt. & 150 to 500 & 182 & 246 & 209.24 & 281 & 293 \\
HBD & 0 to 5 & 3 & 4 & 2 & 4 & 2 \\
HBA & 0 to 10 & 4 & 4 & 5 & 8 & 4 \\
\hline
\end{tabular}

Table 11. Physiochemical Parameters of Proposed lead molecules for PRI2 Riboswitch

\begin{tabular}{lcccccc}
\hline Parameter & $\begin{array}{c}\text { Optimum } \\
\text { range }\end{array}$ & NCI_117268_a & NCI_170621 & NCI_281816_a & NCI_403379 & NCI_13316_b \\
\hline ClogP & -5 to +5 & 1.68 & 0.71 & 4.68 & -0.65 & 4.56 \\
PSA (2D) $\left(\AA^{2}\right)$ & 60 to 140 & 140 & 63.17 & 6.48 & 108 & 45.15 \\
Mol. Wt. & 150 to 500 & 358 & 203 & 356 & 205 & 346 \\
HBD & 0 to 5 & 3 & 1 & 1 & 3 & 2 \\
HBA & 0 to 10 & 7 & 3 & 1 & 5 & 2 \\
\hline
\end{tabular}




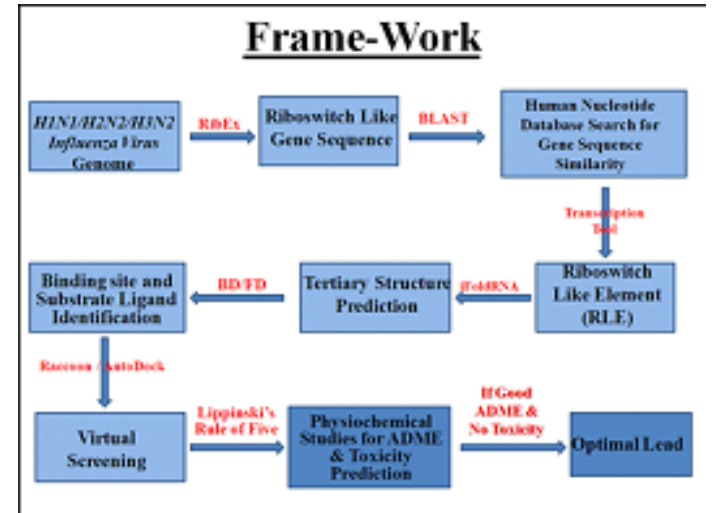

Fig. 1. The flow chart of framework

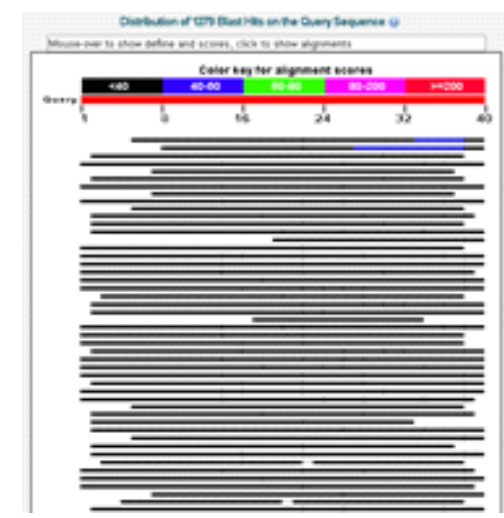

a) predicted three dimensional structure of PRI2 riboswitch of Influenza virus is shown in Fig.7.

The focused docking result of PRI2 riboswitch is tabulated in Table-5.

Docking results obtained by blind docking of both the riboswitches i.e. PRI1 and PRI2 are tabulated in Table-6.

\section{Refined Docking Results}

The results of individual docking of riboswitch PRI1 with Lysine for a repeated number of times are given in Table-7. There was a change in its binding energy from -7.26 to - Kcal/Mol.

Similarly the Arginine is repetitively docked with PRI2 for refinement of their binding

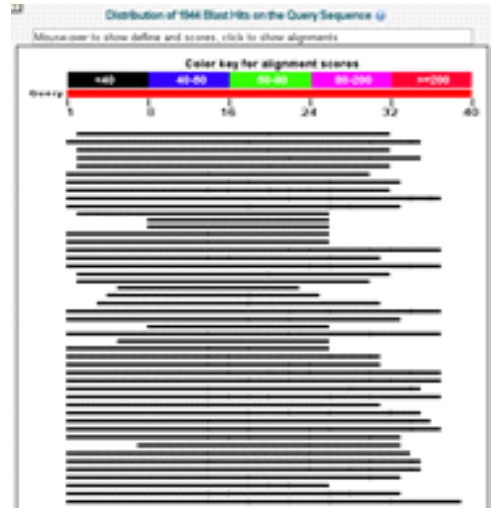

b)

Fig. 2. (A) Similarity of PRI1 gene sequence with Human genome using BLAST. (B) Similarity of PRI2 gene sequence with Human genome using BLAST

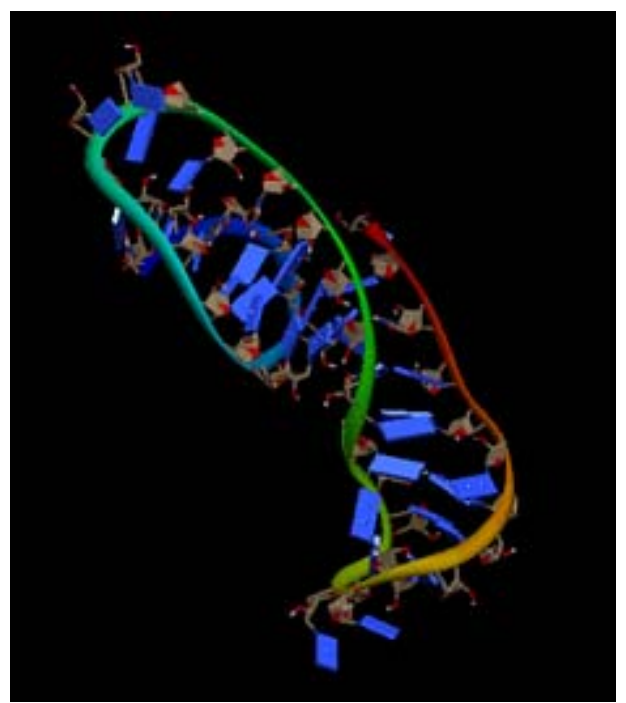

Fig. 3. Predicted structure of riboswitch PRI1 of Influenza virus

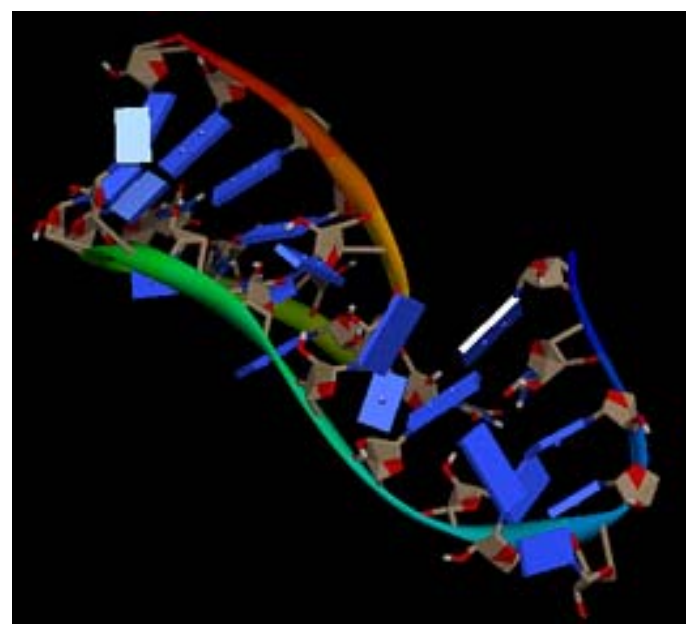

Fig. 4. Predicted structure of riboswitch PRI2 of Influenza virus 


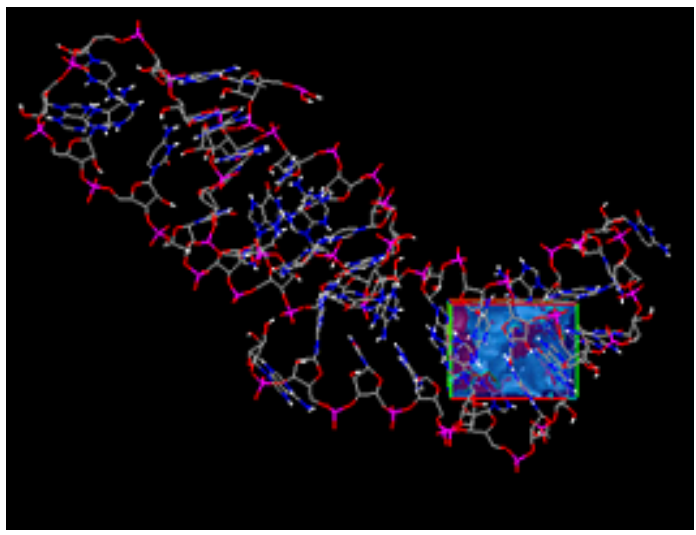

Fig. 5. Grid box used for focused docking of PRI1 riboswitch covering all binding residues involved in binding of ligand.

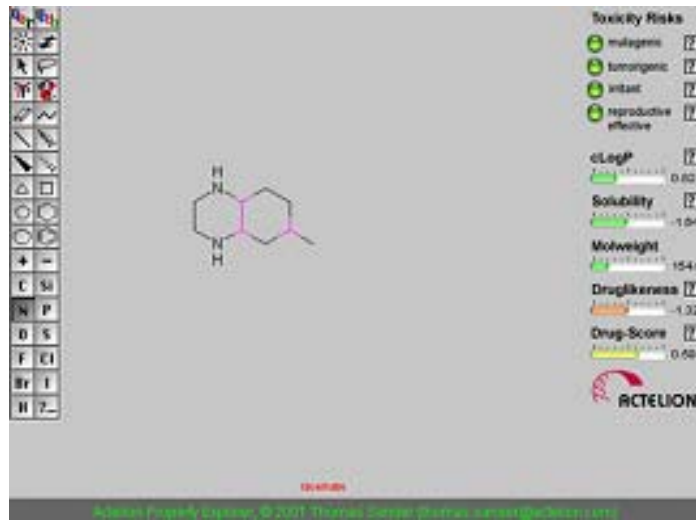

Fig. 7. Toxicity and drug likeness prediction of Lead Compound NCI 81462 a

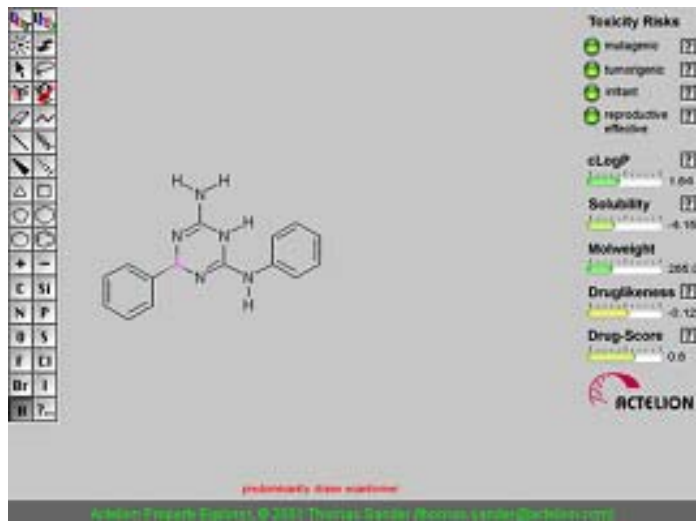

Fig. 9. Toxicity and drug likeness prediction of Lead Compound NCI 113486

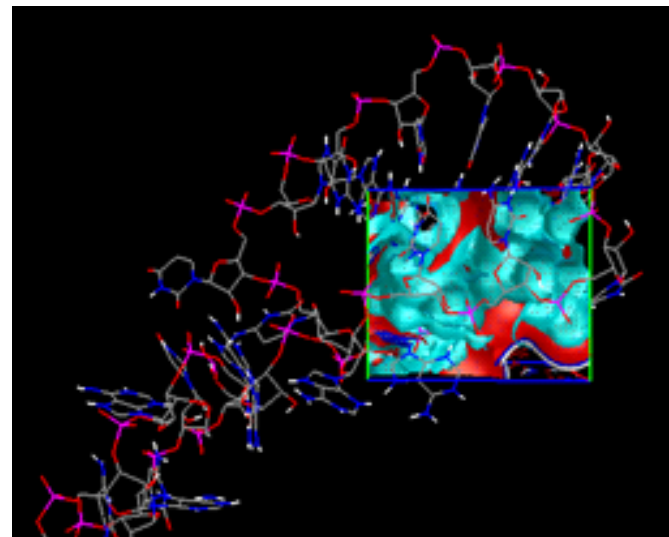

Fig. 6. Grid box used for focused docking of PRI2 riboswitch covering all binding residues involved in binding of ligand

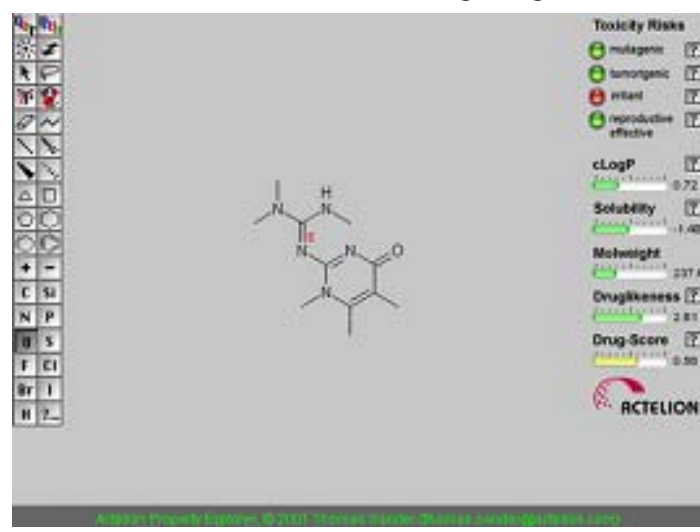

Fig. 8. Toxicity and drug likeness prediction of Lead Compound NCI 168184

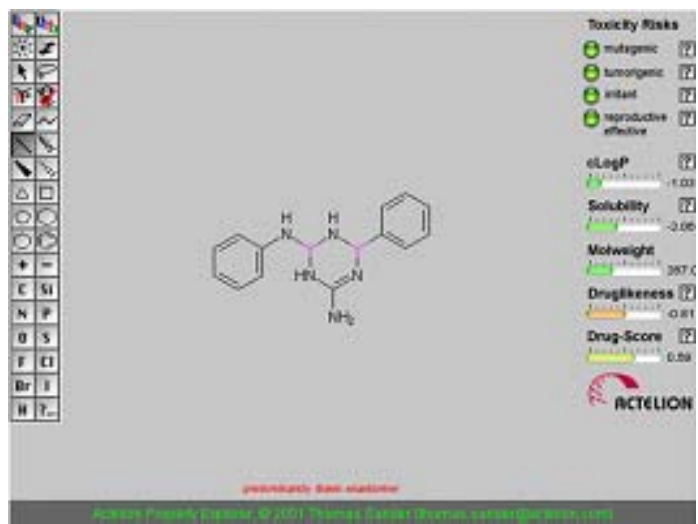

Fig. 10. Toxicity and drug likeness prediction of Lead Compound NCI 207895 


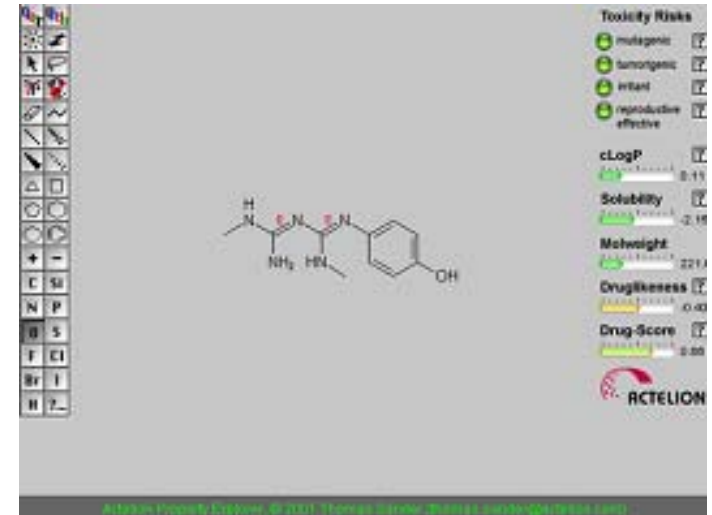

Fig. 11. Toxicity and drug likeness prediction of Lead Compound NCI_3076_a

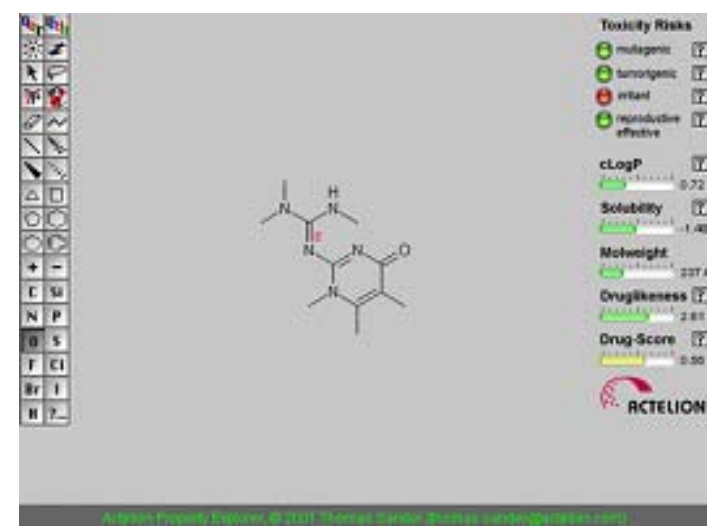

Fig. 13. Toxicity and drug likeness prediction of Lead Compound NCI_170621

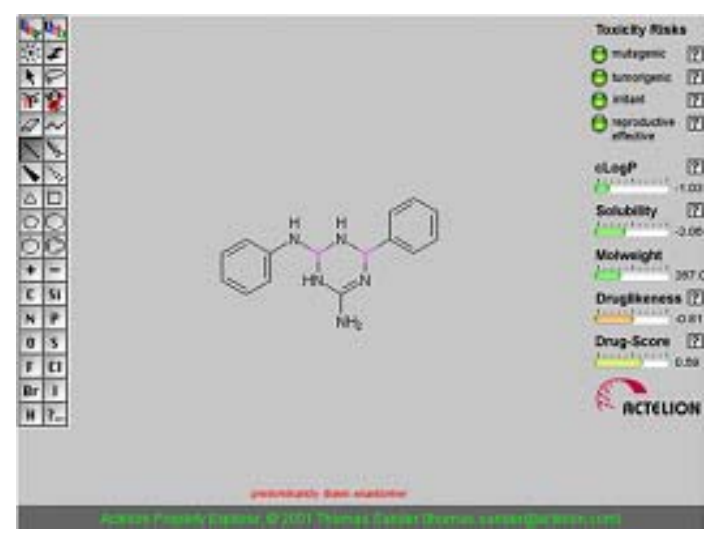

Fig. 15. Toxicity and drug likeness prediction of Lead Compound NCI_403379

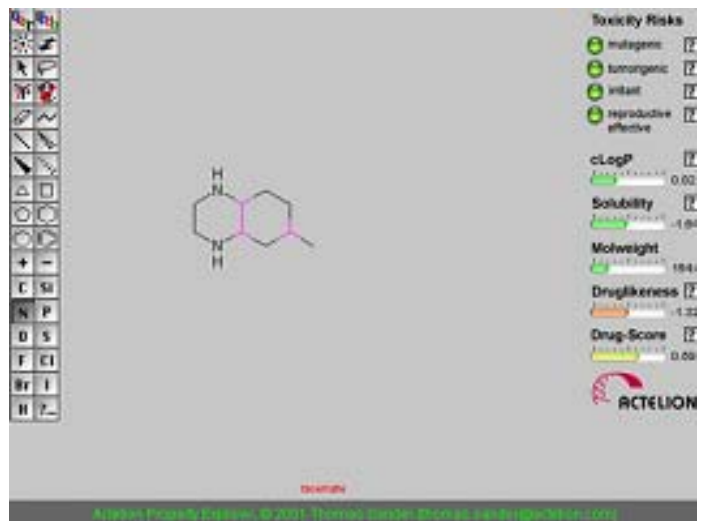

Fig. 12. Toxicity and drug likeness prediction of Lead Compound NCI_117268_a

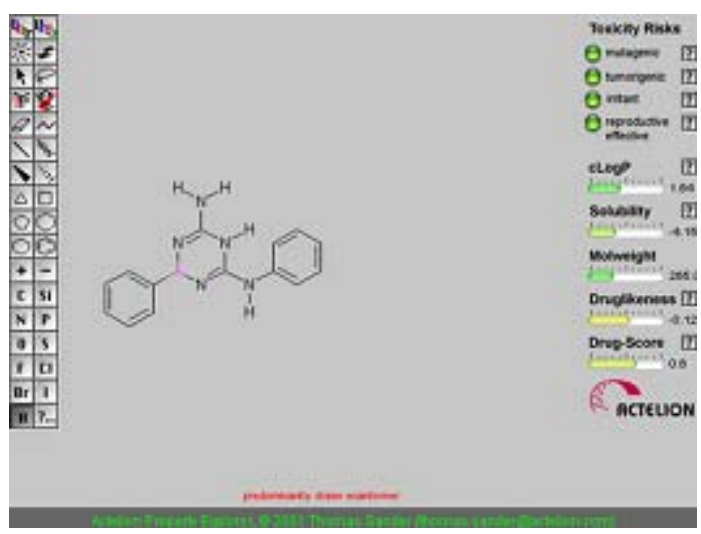

Fig. 14. Toxicity and drug likeness prediction of Lead Compound NCI_281816_a

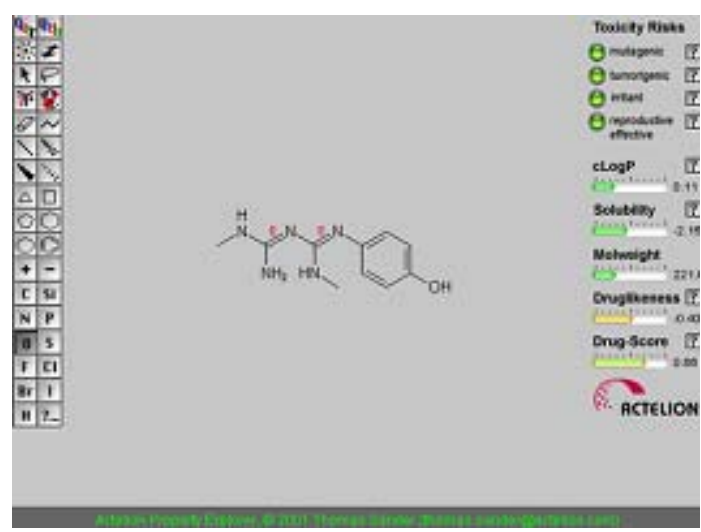

Fig. 16. Toxicity and drug likeness prediction of Lead Compound NCI_13316_b 
energy values. The binding energy for PRI2 riboswitch is refined from -6.37 to $-\mathrm{Kcal} / \mathrm{Mol}$. These refined binding values are tabulated in Table- 7 .

\section{Virtual Screening Results}

The 5 lead molecules for each riboswitch were selected after virtual screening of NCI Diversity Set containing 1541 diverse molecules against all the three predicted Influenza riboswitches PRI1 and PRI2. The binding energy and $\mathrm{Ki}$ value of 5 lead molecules for both of the influenza riboswitches are given in Table- 8 and 9 for each of the predicted riboswitch PRI1 and PRI2 respectively.

(a) The following five lead molecules were obtained for PRI1 riboswitch after the virtual screening: NCI 81462 a, NCI 168184, NCI_113486, NCI_207895 and NCI_3076_a. The binding energy and $\mathrm{Ki}$ value of top 5 lead molecules for PRI1 riboswitches is given in Table- 8 .

(b) The five lead molecules were obtained for PRI2 riboswitch after the virtual screening: NCI_117268_a,NCI_170621,NCI_281816_a, NCI_403379 and NCI_13316_b. The binding energy and Ki value of top 5 lead molecules for PRI1 riboswitches is given in Table-9.

Physicochemical Properties of Screened Lead Compounds

(a) The physicochemical properties of screened top 5 lead molecules for PRI1 influenza riboswitch are tabulated in Table- 10 .

(b) The physicochemical properties of screened top 5 lead molecules for PRI2 Influenza riboswitch is tabulated in Table-11.

\section{ADME \& Toxicity Profiling}

The results of toxicity and ADME prediction of screened top 5 lead compounds for both the riboswitches is computed by using Osiris online program.

(a) The toxicity prediction and drug likeness score for top 5 virtually screened lead molecule for PRI1 riboswitch are shown in Fig. 7 to 11 . The three lead compounds out of top 5 screened leads ZINC19325791, ZINC19362650 and ZINC08652230 passed the toxicity test with a good drug likeness score, while lead ZINC01556940 shows satisfactory drug score but there is a probability of presence of some reproductive effects is shown in the toxicity test. The lead compound ZINC19230120 shows the poor drug likeness score and supposed to be having serious toxic effects such as mutagenicity, irritant nature and reproductive effects.

(b) The toxicity prediction and drug likeness score for top 5 virtually screened lead molecule for PRI2 riboswitch are shown in Fig. 12 to 16 . All the five screened lead compounds ZINC01584497, ZINC03947435, ZINC13597738, ZINC01729525 and ZINC01871223 passed the toxicity test without any toxic effect and satisfactory drug likeness score.

\section{CONCLUSION}

We have been able to perform in-silico prediction of two riboswitches for H1N1, H2N2 and $\mathrm{H} 3 \mathrm{~N} 2$ strains of Influenza A. The in-silico approach has further been successfully used to predict binding sites and specific ligands for these strains of influenza and lead molecules which inhibit these riboswitches. In all the three strains of influenza Lysine is found to be the most efficient ligand.

Thus, we conclude that in-silico approach can be used for prediction of riboswitches, their binding sites, specific ligands, and their potential inhibitors.

The inhibitors predicted are free from the side effects of the antiviral drugs and have remote chances of developing resistance. In all such studies can be useful for developing novel drugs for infectious disease like influenza.

\section{ACKNOWLEDGMENTS}

Authors acknowledge Department of Mathematics, Bioinformatics \& Computer Application, Maulana Azad National Institute of Technology (M.A.N.I.T.), Bhopal, for providing the facilities to carry out the project under the immense guidance of Dr. K.R. Pardasani (Professor, Department of Mathematics and Former Head of Department of Mathematics, Bioinformatics \& Computer Application). 


\section{REFERENCES}

1. Somdutt M, Kamal RP, Riboswitch as a target for Streptococcus pneumoniae. Online $J$ Bioinformatics 2012; 13(2): 285-313

2. Hall CB., The spread of influenza and other respiratory viruses: complexities and conjectures. Clin Infect Dis 2007; 45(3):353359. doi:10.1086/519433

3. Brankston G, Gitterman L, Hirji Z, Lemieux C, Gardam M., Transmission of influenza A in human beings. Lancet Infect Dis 2007; 7(4):257265. doi:10.1016/S1473-3099(07)70029-4

4. Tellier R., Review of aerosol transmission of influenza A virus. Emerg Infect Dis 2006; 12(11): 1657

5. Bell D., Non-pharmaceutical interventions for pandemic influenza, international measures. Emerg Infect Dis 2006; 12(1):81-87. doi:10.3201/eid1201.051370

6. Klontz KC, Hynes NA, Gunn RA, Wilder MH, Harmon MW, Kendal AP., An outbreak of influenza A/Taiwan/1/86 (H1N1) infections at a naval base and its association with airplane travel. Am J Epidemiol 1989; 129(2):341-348

7. Moser MR, Bender TR, Margolis HS, Noble GR, Kendal AP, Ritter DG., An outbreak of influenza aboard a commercial airliner. Am J Epidemiol 1979; 110(1):1-6

8. Glezen WP, Couch RB, MacLean RA, Payne A, Baird JN, Vallbona C, Tristan M, Byrd N., Interpandemic influenza in the Houston area, 1978; 1974-76. New Engl J Med 298 (11):587592. doi:10.1056/NEJM197803162981103

9. Monto AS, Kioumehr F., The Tecumseh study of respiratory illness IX. Occurrence of influenza in the community, 1966-1971. Am J Epidemiol 1975; 102(6):553-563

10. Walsh EE, Cox C, Falsey AR., Clinical features of influenza A virus infection in older hospitalized persons. J Am Geriatr Soc 2002; 50(9):1498-1503. doi:10.1046/j.1532-5415. 2002. 50404.X

11. Keren R, Zaoutis TE, Bridges CB, Herrera G, Watson BM, Wheeler AB, Licht DJ, Luan XQ, Coffin SE., Neurological and neuromuscular disease as a risk factor for respiratory failure in children hospitalized with influenza infection. JAMA-J Am Med Assoc 2005; 294(17): 21882194. doi:10.1001/jama.294.17.2188

12. Bright RA, Medina M-j, Xu X, Perez-Oronoz G, Wallis TR, Davis XM, Povinelli L, Cox NJ, Klimov AI., Incidence of adamantane resistance among influenza A (H3N2) viruses isolated worldwide from 1994 to 2005: a cause for concern. Lancet 2005; 366(9492):1175-1181. doi:10.1016/s0140-6736(05)67338-2

13. Updated Interim Recommendations for the Use of Antiviral Medications in the Treatment and Prevention of Influenza for the 2009-2010 Season. 2009, http://www.cdc.gov/h1n1flu/ recommendations.htm.

14. Bautista E, Chotpitayasunondh T, Gao Z, Harper SA, Shaw M, Uyeki TM, Zaki SR, Hayden FG, Hui DS, Kettner JD., Clinical aspects of pandemic 2009 influenza A (H1N1) virus infection, 2010. doi:10.1056/ NEJMra1000449

15. Harper SA, Bradley JS, Englund JA, File TM, Gravenstein S, Hayden FG, McGeer AJ, Neuzil KM, Pavia AT, Tapper ML., Seasonal influenza in adults and children-diagnosis, treatment, chemoprophylaxis, and institutional outbreak management: clinical practice guidelines of the Infectious Diseases Society of America. Clin Infect Dis 2009; 48(8):1003-1032. doi:10.1086/ 598513

16. Gubareva LV, Matrosovich MN, Brenner MK, Bethell RC, Webster RG., Evidence for zanamivir resistance in an immunocompromised child infected with influenza B virus. J Infect Dis 1998; 178(5):1257-1262. doi:10.1086/314440

17. Stephenson I, Democratis J, Lackenby A, McNally T, Smith J, Pareek M, Ellis J, Bermingham A, Nicholson K, Zambon M, Neuraminidase inhibitor resistance after oseltamivir treatment of acute influenza A and B in children. Clin Infect Dis 2009; 48(4):389396. doi:10.1086/596311

18. Gubareva LV, Kaiser L, Matrosovich MN, SooHoo Y, Hayden FG, Selection of influenza virus mutants in experimentally infected volunteers treated with oseltamivir. J Infect Dis 2001; 183(4): 523-531. doi:10.1110/ps.0202302

19. Barnett J, Cadman A, Gor D, Dempsey M, Walters M, Candlin A, Tisdale M, Morley P, Owens I, Fenton R, Zanamivir susceptibility monitoring and characterization of influenza virus clinical isolates obtained during phase II clinical efficacy studies. Antimicrob Agents $\mathrm{CH} 2000$; 44(1): 78-87. doi:10.1128/AAC.44.1.7887.2000

20. Kiso M, Mitamura K, Sakai-Tagawa Y, Shiraishi K, Kawakami C, Kimura K, Hayden FG, Sugaya $\mathrm{N}$, Kawaoka Y, Resistant influenza A viruses in children treated with oseltamivir: descriptive study. Lancet 2004; 364(9436):759-765. doi:10.1016/s0140-6736(04)16934-1

21. Ison MG, Gubareva LV, Atmar RL, Treanor J, Hayden FG, Recovery of drug-resistant influenza virus from immunocompromised patients: a case 
series. J Infect Dis 2006; 193(6):760-764. doi:10.1086/500465

22. Farias JA, Fernández A, Monteverde E, Vidal N, Arias P, Montes MJ, Rodríguez G, Allasia M, Ratto ME, Jaén R, Critically ill infants and children with influenza A (H1N1) in pediatric intensive care units in Argentina. Intens Care Med 2010; 36(6):1015-1022. doi:10.1007/ s00134-010-1853-1

23. Karie S, Launay-Vacher V, Janus N, Izzedine H, Deray G., Pharmacokinetics and dosage adjustment of oseltamivir and zanamivir in patients with renal failure. Nephrol Dial Transpl 2006; 21(12): 3606-3608. doi:10.1093/ndt/ gfl345

24. Mandal M, Breaker RR, Gene regulation by riboswitches. Nat Rev Mol Cell Bio 2004; 5(6): 451-463. doi:10.1038/nrm1403

25. Cheah MT, Wachter A, Sudarsan N, Breaker RR, Control of alternative RNA splicing and gene expression by eukaryotic riboswitches. Nature 2007; 447 (7143):497-500. doi:10.1038/ nature 05769

26. Wachter A, Tunc-Ozdemir M, Grove BC, Green PJ, Shintani DK, Breaker RR, Riboswitch control of gene expression in plants by splicing and alternative 32 end processing of mRNAs. Plant Cell 2007; 19(11):3437-3450

27. Blount KF, Wang JX, Lim J, Sudarsan N, Breaker $\mathrm{RR}$, Antibacterial lysine analogs that target lysine riboswitches. Nat Chem Biol 2006; 3(1):44-49. doi:10.1038/nchembio842

28. Baird NJ, Kulshina N, D'Amare ARF, Riboswitch function: flipping the switch or tuning the dimmer? RNA Biol 2010; 7(3):328332. doi:10.4161/rna.7.3.11932

29. Iorga B, Herlem D, Barré E, Guillou C, Acetylcholine nicotinic receptors: finding the putative binding site of allosteric modulators using the "blind docking" approach. J Mol Model 2006; 12(3):366-372. doi:10.1007/s00894-0050057-z

30. Morris GM, Huey R, Lindstrom W, Sanner MF, Belew RK, Goodsell DS, Olson AJ, AutoDock4 and AutoDockTools4: Automated docking with selective receptor flexibility. J Comput Chem 2009; 30(16):2785-2791. doi:10.1002/jcc.21256

31. Famulok M, Hartig JS, Mayer G., Functional aptamers and aptazymes in biotechnology, diagnostics, and therapy. Chem Rev 2007; 107(9): 3715-3743. doi:10.1021/cr0306743

32. Bikádi Z, Hazai E, Zsila F, Lockwood SF, Molecular modeling of non-covalent binding of homochiral $(3<\mathrm{i}>\mathrm{S}</ \mathrm{i}>, 32<\mathrm{i}>\mathrm{S}</ \mathrm{i}>)$ astaxanthin to matrix metalloproteinase-13 (MMP-13). Bioorgan Med Chem 2006; 14(16): 5451-5458. doi:10.1016/j.bmc.2006.04.047

33. Hetényi C, van der Spoel D, Efficient docking of peptides to proteins without prior knowledge of the binding site. Protein Sci 2002; 11(7):17291737. doi:10.1110/ps.0202302. 